\title{
UNIVERSAL CENTRAL EXTENSIONS IN SEMI-ABELIAN CATEGORIES
}

\author{
JOSÉ MANUEL CASAS AND TIM VAN DER LINDEN
}

\begin{abstract}
Basing ourselves on Janelidze and Kelly's general notion of central extension, we study universal central extensions in the context of semi-abelian categories. We consider a new fundamental condition on composition of central extensions and give examples of categories which do, or do not, satisfy this condition.
\end{abstract}

\section{INTRODUCTION}

In this article we explain how, using Janelidze and Kelly's general notion of central extension [19], the classical theory of universal central extensions may be considered in the context of semi-abelian categories [20. Our main point is that, while most of the results valid for groups and Lie algebras (see, for instance, [23, 24]) generalise without any difficulty to extensions, central with respect to a chosen Birkhoff subcategory $\mathcal{B}$ of a semi-abelian category $\mathcal{A}$, this setting turns out to be too weak for some of the most basic results, valid in the classical examples, to hold - even when $\mathcal{B}=\operatorname{Ab}(\mathcal{A})$ is determined by the abelian objects in $\mathcal{A}$.

We have to impose an additional condition which we chose to call the universal central extension condition (UCE), asking that for $\operatorname{Ab}(\mathcal{A})$-central extensions $f: B \rightarrow A$ and $g: C \rightarrow B$ also the composite extension $f \circ g$ is $\mathrm{Ab}(\mathcal{A})$-central, as soon as $B$ is an $\operatorname{Ab}(\mathcal{A})$-perfect object. (Recall that in general, central extensions need not compose.) Under condition (UCE) and, as it turns out, only then, standard recognition results such as Theorem 4.4 hold. Furthermore, as Example 5.2 shows, condition (UCE) is not automatic: there exist semi-abelian categories which do not satisfy it. This immediately gives rise to the following question, which is not yet fully answered in the present paper: When does condition (UCE) hold? We can give examples and counterexamples, but thus far there is no elementary characterisation. This problem - of finding good minimal hypotheses for condition (UCE) - is the subject of current work-in-progress [16].

\section{BASIC DEFINITIONS AND FIRST RESUlTS}

1.1. Semi-abelian categories. A category is semi-abelian when it is pointed, Barr exact and Bourn protomodular with binary coproducts [20]. We recall that a pointed and regular category is Bourn protomodular [5] if and only if the (Regular) Short Five Lemma holds: this means that for any commutative

2010 Mathematics Subject Classification. 17A32, 18B99, 18E99, 18G50, 20J05.

Key words and phrases. Categorical Galois theory; semi-abelian category; homology; Baer invariant; universal central extension.

The first author's research was supported by Ministerio de Ciencia e Innovación (grant number MTM2009-14464-C02-02, includes European FEDER support) and by Xunta de Galicia (grant number Incite09 $207215 \mathrm{PR}$ ). The second author works as chargé de recherches for Fonds de la Recherche Scientifique-FNRS. His research was supported by Centro de Matemática da Universidade de Coimbra and by Fundação para a Ciência e a Tecnologia (grant number SFRH/BPD/38797/2007). We thank the University of Coimbra, the University of Vigo and the Banff International Research Station for their kind hospitality. 
diagram such as (A) below where $f$ and $f^{\prime}$ are regular epimorphisms, $k$ and $a$ being isomorphisms implies that $b$ is an isomorphism. It is well known that all varieties of $\Omega$-groups [17] are semi-abelian categories.

Lemma 1.2. [5, 6] Consider a morphism of short exact sequences

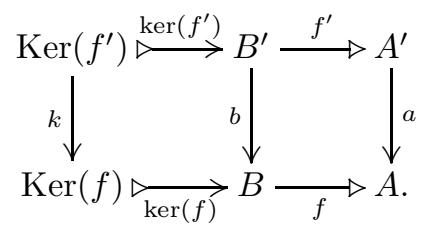

(1) The right hand side square $f \circ b=a \circ f^{\prime}$ is a pullback iff $k$ is an isomorphism.

(2) The left hand side square $\operatorname{ker}(f) \circ k=b \circ \operatorname{ker}\left(f^{\prime}\right)$ is a pullback iff a is mono.

The first statement implies that any pullback square between regular epimorphisms (that is, any square $f \circ b=a \circ f^{\prime}$ as in (A) ) is a pushout. It is also well known that the regular image of a kernel is a kernel [20]. In any semi-abelian category, classical homological lemma's such as the Snake Lemma and the $3 \times 3$ Lemma are valid; for further details and many other results we refer the reader to [20, 4].

1.3. Birkhoff subcategories. The notion of central extension introduced in [19] is defined with respect to a chosen subcategory $\mathcal{B}$ of the base category $\mathcal{A}$ : a Birkhoff subcategory $\mathcal{B}$ of a semi-abelian category $\mathcal{A}$ is a full and reflective subcategory, closed under subobjects and regular quotients. We denote the left adjoint by $I: \mathcal{A} \rightarrow \mathcal{B}$ and write the components of its unit $\eta_{A}: A \rightarrow I(A)$. A Birkhoff subcategory of a variety of universal algebras is the same thing as a subvariety. Throughout the text, we fix a Birkhoff subcategory $\mathcal{B}$ of a semi-abelian category $\mathcal{A}$.

1.4. Extensions and central extensions. An extension in $\mathcal{A}$ is a regular epimorphism. A morphism of extensions is a commutative square between them, and thus we obtain the category $\operatorname{Ext}(\mathcal{A})$ of extensions in $\mathcal{A}$.

With respect to $\mathcal{B} \subseteq \mathcal{A}$, there are notions of trivial, normal and central extension. An extension $f: B \rightarrow A$ in $\mathcal{A}$ is said to be trivial if and only if the induced square

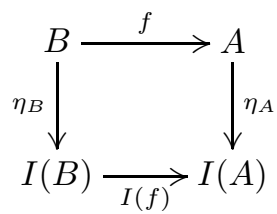

is a pullback. The extension $f$ is normal if and only if one of the projections $f_{0}, f_{1}$ in the kernel pair $\left(B \times{ }_{A} B, f_{0}, f_{1}\right)$ of $f$ is trivial. Finally, $f$ is said to be central if and only if there exists an extension $g: C \rightarrow A$ such that the pullback $g^{*}(f)$ of $f$ along $g$ is trivial.

Clearly, every normal extension is central; in the present context, the converse also holds, and thus the concepts of normal and central extension coincide. Furthermore, a split epimorphism is a trivial extension if and only if it is central [19, Theorem 4.8]. Finally, central extensions are pullback-stable [19, Proposition 4.3].

Since we shall often be considering several Birkhoff subcategories of a given category at the same time, we usually indicate which one we mean by prefixing, as in "B-central", " $I$-trivial", etc.

1.5. Perfect objects. An object $P$ of $\mathcal{A}$ is called perfect when $I(P)$ is the zero object 0 of $\mathcal{B}$. If $f: B \rightarrow A$ is an extension and $B$ is $\mathcal{B}$-perfect then so is $A$, because the reflector $I$ preserves regular epimorphisms, and a regular quotient of zero is zero. 
Lemma 1.6. Let $P$ be a $\mathcal{B}$-perfect object and $f: B \rightarrow A$ an extension.

(1) If $f$ is $\mathcal{B}$-trivial then the map

$$
\operatorname{Hom}(P, f)=f \circ(-): \operatorname{Hom}(P, B) \rightarrow \operatorname{Hom}(P, A)
$$

is a bijection;

(2) if $f$ is $\mathcal{B}$-central then $\operatorname{Hom}(P, f)$ is an injection.

If $\operatorname{Hom}(P, f)$ is an injection for every $\mathcal{B}$-trivial extension $f$ then $P$ is $\mathcal{B}$-perfect.

Proof. The extension $f$ being $\mathcal{B}$-trivial means that the square $(\mathbf{B})$ is a pullback. If $b_{0}, b_{1}: P \rightarrow B$ are morphisms such that $f \circ b_{0}=f \circ b_{1}$, then $b_{0}$ is equal to $b_{1}$ by the uniqueness in the universal property of this pullback: indeed also $\eta_{B} \circ b_{0}=$ $I\left(b_{0}\right) \circ \eta_{P}=0=I\left(b_{1}\right) \circ \eta_{P}=\eta_{B} \circ b_{1}$. Thus we see that $\operatorname{Hom}(P, f)$ is injective. This map is also surjective, since any morphism $a: P \rightarrow A$ is such that $\eta_{A^{\circ}} a=I(a) \circ \eta_{P}=$ $0=I(f) \circ 0$ and thus induces a morphism $b: P \rightarrow B$ for which $f \circ b=a$.

Statement 2 follows from 1 because the functor $\operatorname{Hom}(P,-)$ preserves kernel pairs, and a map is an injection if and only if its kernel pair projections are bijections.

As to the converse: the morphism $!_{I(P)}: I(P) \rightarrow 0$ is a $\mathcal{B}$-trivial extension; since $!_{I(P)}{ }^{\circ} \eta_{P}=0=!_{I(P)} \circ$, the assumption implies that $\eta_{P}$ is zero, which means that $P$ is $\mathcal{B}$-perfect.

1.7. Universal central extensions. For any object $A$ of $\mathcal{A}$, let $\operatorname{Centr}_{\mathcal{B}}(A)$ or $\operatorname{Centr}_{I}(A)$ denote the category of all $\mathcal{B}$-central extensions of $A$ : the full subcategory of the slice category $(\mathcal{A} \downarrow A)$ determined by the central extensions. A (weakly) initial object of this category $\operatorname{Centr}_{\mathcal{B}}(A)$ is called a (weakly) universal central extension of $A$. A $\mathcal{B}$-central extension $u: U \rightarrow A$ is weakly universal when for every $\mathcal{B}$-central extension $f: B \rightarrow A$ there exists a morphism $\bar{f}$ from $u$ to $f$, that is, such that $f \circ \bar{f}=u$. Furthermore, $u$ is universal when this induced morphism $\bar{f}$ is unique. Note also that, up to isomorphism, an object admits at most one universal $\mathcal{B}$-central extension.

Lemma 1.8. If $u: U \rightarrow A$ is a universal $\mathcal{B}$-central extension then the objects $U$ and $A$ are $\mathcal{B}$-perfect.

Proof. Since the first projection $\pi_{A}: A \times I(U) \rightarrow A$ is a trivial extension, by [1.4 it is central. By the hypothesis that $u$ is universal, there exists just one morphism $\langle u, v\rangle: U \rightarrow A \times I(U)$ such that $\pi_{A} \circ\langle u, v\rangle=u$. But then $0: U \rightarrow I(U)$ is equal to $\eta_{U}: U \rightarrow I(U)$, and $I(U)=0$. Since a regular quotient of a perfect object is perfect, this implies that both $U$ and $A$ are $\mathcal{B}$-perfect.

Proposition 1.9. Let $\mathcal{A}$ be a semi-abelian category and $\mathcal{B}$ a Birkhoff subcategory of $\mathcal{A}$. Let $u: U \rightarrow A$ be a $\mathcal{B}$-central extension. Between the following conditions, the implications $1 \Leftrightarrow 2 \Leftrightarrow 3 \Rightarrow 4 \Leftrightarrow 5$ hold:

(1) $U$ is $\mathcal{B}$-perfect and every $\mathcal{B}$-central extension of $U$ splits;

(2) $U$ is $\mathcal{B}$-perfect and projective with respect to all $\mathcal{B}$-central extensions;

(3) for every $\mathcal{B}$-central extension $f: B \rightarrow A$, the map

$$
\operatorname{Hom}(U, f): \operatorname{Hom}(U, B) \rightarrow \operatorname{Hom}(U, A)
$$

is a bijection;

(4) $U$ is $\mathcal{B}$-perfect and $u$ is a weakly universal $\mathcal{B}$-central extension;

(5) $u$ is a universal $\mathcal{B}$-central extension.

Proof. Suppose that 1 holds. To prove 2, let $f: B \rightarrow A$ be a $\mathcal{B}$-central extension and $g: U \rightarrow A$ a morphism. Then the pullback $g^{*}(f): \bar{B} \rightarrow U$ of $f$ along $g$ is still $\mathcal{B}$-central; hence $g^{*}(f)$ admits a splitting $s: U \rightarrow \bar{B}$, and $\left(f^{*}(g)\right) \circ s$ is the required morphism $g \rightarrow f$. Conversely, given a $\mathcal{B}$-central extension $f: B \rightarrow U$, the projectivity of $U$ yields a morphism $s: U \rightarrow B$ such that $f \circ s=1_{U}$. 
Conditions 2 and 3 are equivalent by Lemma 1.6 .

Condition 3 implies condition 5: given a $\mathcal{B}$-central extension $f: B \rightarrow A$ of $A$, there exists a unique morphism $\bar{f}: U \rightarrow B$ that satisfies $f \circ \bar{f}=u$.

Finally, 4 and 5 are equivalent by Lemma 1.6 and Lemma 1.8 .

Remark 1.10. To prove that condition 4 implies 3 we would require $U$ itself to admit a universal $\mathcal{B}$-central extension, which need not be the case in the present context. In fact, even if such a universal $\mathcal{B}$-central extension of $U$ does exist, then the above five conditions may or may not be equivalent: see Section 4 .

\section{Constructing universal Central extensions}

Our aim is now to prove that every perfect object admits a universal central extension. To do so, we need to assume that the surrounding category has enough projectives: they will give us weakly universal central extensions.

2.1. Commutators and centralisation. The kernel $\mu$ of the unit $\eta$ of $I: \mathcal{A} \rightarrow \mathcal{B}$ gives rise to a "zero-dimensional" commutator: for any object $A$ of $\mathcal{A}$, the bottom row in $(\mathbf{C}$ is a short exact sequence in $\mathcal{A}$; hence $A$ is an object of $\mathcal{B}$ if and only if $[A, A]_{\mathcal{B}}=0$. On the other hand, an object $A$ of $\mathcal{A}$ is $\mathcal{B}$-perfect precisely when $[A, A]_{\mathcal{B}}=A$. This construction defines a functor $[-,-]_{\mathcal{B}}: \mathcal{A} \rightarrow \mathcal{A}$ and a natural transformation $\mu:[-,-]_{\mathcal{B}} \Rightarrow 1_{\mathcal{A}}$. The functor $[-,-]_{\mathcal{B}}$ preserves regular epimorphisms; we recall the argument. Given a regular epimorphism $f: B \rightarrow A$, by the Birkhoff property, the induced square of regular epimorphisms on the right

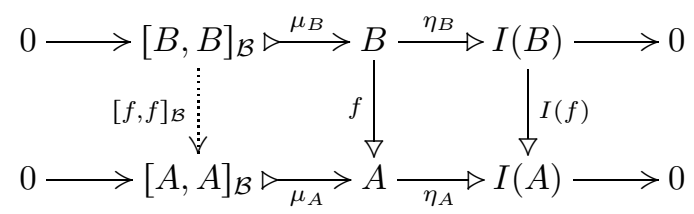

is a pushout - which is equivalent to $[f, f]_{\mathcal{B}}$ being regular epic [12, Corollary 5.7].

Lemma 1.2 implies that an extension $f: B \rightarrow A$ is $\mathcal{B}$-central if and only if either one of the morphisms $\left[f_{0}, f_{0}\right]_{\mathcal{B}},\left[f_{1}, f_{1}\right]_{\mathcal{B}}$ is an isomorphism, which, because they have a common splitting, happens exactly when they coincide, $\left[f_{0}, f_{0}\right]_{\mathcal{B}}=\left[f_{1}, f_{1}\right]_{\mathcal{B}}$. Hence the kernel $[K, B]_{\mathcal{B}}$ of $\left[f_{0}, f_{0}\right]_{\mathcal{B}}$ measures how far $f$ is from being central: indeed, $f$ is $\mathcal{B}$-central if and only if $[K, B]_{\mathcal{B}}$ is zero.

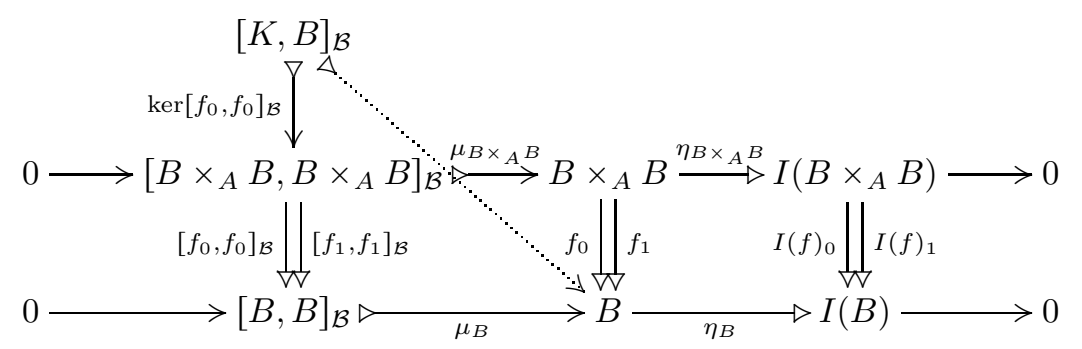

Remark 2.2. This explains, for instance, why a sub-extension of a central extension is central. It is worth recalling here that a morphism of extensions $(b, a)$

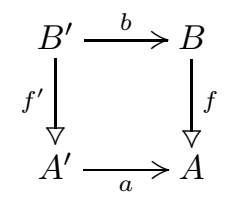

is a monomorphism if and only if $b$ is. 
The "one-dimensional" commutator $[K, B]_{\mathcal{B}}$ may be considered as a normal subobject of $B$ via the composite $\mu_{B} \circ\left[f_{1}, f_{1}\right]_{\mathcal{B}} \circ \operatorname{ker}\left[f_{0}, f_{0}\right]_{\mathcal{B}}:[K, B]_{\mathcal{B}} \rightarrow B$ (see the diagram above). Thus we obtain the left adjoint $I_{1}: \operatorname{Ext}(\mathcal{A}) \rightarrow \operatorname{CExt}_{\mathcal{B}}(\mathcal{A})$, where $\operatorname{CExt}_{\mathcal{B}}(\mathcal{A})$ is the full reflective subcategory of $\operatorname{Ext}(\mathcal{A})$ determined by the $\mathcal{B}$-central extensions. Given an extension $f: B \rightarrow A$ with kernel $K$, its centralisation $I_{1}(f): B /[K, B]_{\mathcal{B}} \rightarrow A$ is obtained through the diagram with exact rows

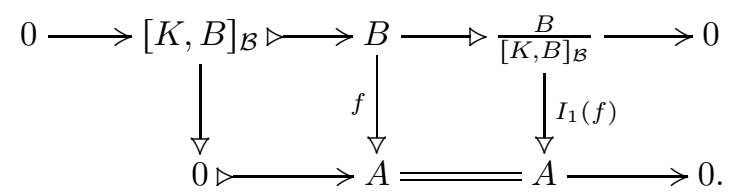

2.3. Existence of a weakly universal central extension. We say that $\mathcal{A}$ has weakly universal central extensions (for some Birkhoff subcategory $\mathcal{B}$ of $\mathcal{A}$ ) when every object of $\mathcal{A}$ admits a weakly universal $\mathcal{B}$-central extension. This happens, for instance, when $\mathcal{A}$ has enough (regular) projectives, so that for any object $A$ of $\mathcal{A}$, there exists a regular epimorphism $f: B \rightarrow A$ with $B$ projective, a (projective) presentation of $A$.

Lemma 2.4. If the category $\mathcal{A}$ is semi-abelian with enough projectives then it has weakly universal central extensions for any Birkhoff subcategory $\mathcal{B}$.

Proof. Given an object $A$ of $\mathcal{A}$, the category $\operatorname{Centr}_{\mathcal{B}}(A)$ has a weakly initial object: given a projective presentation $f: B \rightarrow A$ with kernel $K$, its centralisation $I_{1}(f): B /[K, B]_{\mathcal{B}} \rightarrow A$ is weakly initial. Indeed, any $\mathcal{B}$-central extension $g: C \rightarrow A$ induces a morphism $I_{1}(f) \rightarrow g$ in $\operatorname{Centr}_{\mathcal{B}}(A)$, because the object $B$ is projective.

2.5. Baer invariants. Let $A$ be an object of $\mathcal{A}$ and $f: B \rightarrow A$ a projective presentation with kernel $K$. The induced objects

$$
\frac{[B, B]_{\mathcal{B}}}{[K, B]_{\mathcal{B}}} \quad \text { and } \quad \frac{K \wedge[B, B]_{\mathcal{B}}}{[K, B]_{\mathcal{B}}}
$$

are independent of the chosen projective presentation of $A$ as explained for instance in [12. The object $\left(K \wedge[B, B]_{\mathcal{B}}\right) /[K, B]_{\mathcal{B}}$ is called (the Hopf formula for) the second homology object of $A$ (with coefficients in $\mathcal{B}$ ) and is written $\mathrm{H}_{2}(A, I)$. We write $\mathrm{U}(A, I)$ for the object $[B, B]_{\mathcal{B}} /[K, B]_{\mathcal{B}}$ and $\mathrm{H}_{1}(A, I)$ for $I(A)$.

The objects $\mathrm{H}_{2}(A, I)$ and $\mathrm{H}_{1}(A, I)$ are genuine homology objects: if $\mathcal{A}$ is a semiabelian monadic category then they may be computed using comonadic homology as in [13], and in any case, they fit into the homology theory worked out in [11].

The Baer invariants from 2.5 may also be considered for all weakly universal $\mathcal{B}$-central extensions of an object $A$. Since, for any weakly universal $\mathcal{B}$-central extension $f: B \rightarrow A$ with kernel $K$, the commutator $[K, B]_{\mathcal{B}}$ is zero, this implies that the objects

$$
[B, B]_{\mathcal{B}} \quad \text { and } \quad K \wedge[B, B]_{\mathcal{B}}
$$

are independent of the chosen weakly universal central extension of $A$. (Here, as in [18, the Hopf formula becomes $\mathrm{H}_{2}(A, I)=K \wedge[B, B]_{\mathcal{B}}$. Also note that $\left.\mathrm{U}(A, I)=[B, B]_{\mathcal{B}}.\right)$

2.6. The perfect subobject. When there are weakly universal central extensions, any central extension of a perfect object contains a subobject with a perfect domain. We prove this in two steps: first for weakly universal central extensions, then in general. This implies that any perfect object admits a universal central extension when weakly universal central extensions exist - a general version of Proposition 4.1 in 15 . 
Lemma 2.7. Suppose $\mathcal{A}$ is a semi-abelian category with a Birkhoff subcategory $\mathcal{B}$. Then any weakly universal $\mathcal{B}$-central extension of a $\mathcal{B}$-perfect object contains a subobject with a $\mathcal{B}$-perfect domain.

Proof. Let $f: B \rightarrow A$ be a weakly universal $\mathcal{B}$-central extension of a $\mathcal{B}$-perfect object $A$. Since $\mu_{A}$ is an isomorphism and $[f, f]_{\mathcal{B}}$ is a regular epimorphism, the morphism $f \circ \mu_{B}=\mu_{A} \circ[f, f]_{\mathcal{B}}$ in the induced diagram with exact rows

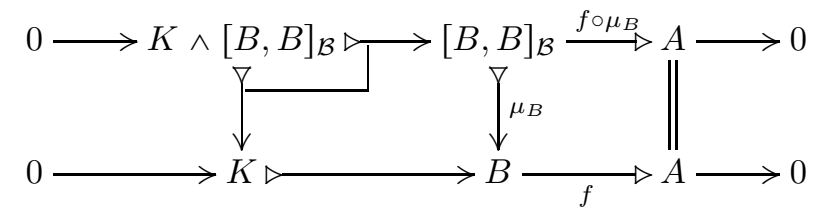

is also a regular epimorphism. The extension $f \circ \mu_{B}$ is $\mathcal{B}$-central as a subobject of the $\mathcal{B}$-central extension $f$; its weak universality is clear. By[2.5 the object $[B, B]_{\mathcal{B}}$ is $\mathcal{B}$-perfect, because the $\mathcal{B}$-central extensions $f \circ \mu_{B}$ and $f$ are both weakly universal, so that $[B, B]_{\mathcal{B}} \cong\left[[B, B]_{\mathcal{B}},[B, B]_{\mathcal{B}}\right]_{\mathcal{B}}$.

Lemma 2.8. Let $\mathcal{A}$ be a semi-abelian category with weakly universal central extensions for a Birkhoff subcategory $\mathcal{B}$ of $\mathcal{A}$. If $f: B \rightarrow A$ is a $\mathcal{B}$-central extension of a $\mathcal{B}$-perfect object $A$, then $[B, B]_{\mathcal{B}}$ is also $\mathcal{B}$-perfect.

Proof. The object $B$ admits a weakly universal $\mathcal{B}$-central extension $v: V \rightarrow B$; then the centralisation $w: W \rightarrow A$ of the resulting composite $f \circ v$ is a weakly universal $\mathcal{B}$-central extension. Indeed, given any $\mathcal{B}$-central extension $g: C \rightarrow A$, there is a factorisation $\overline{f^{*}(g)}: V \rightarrow B \times{ }_{A} C$ of $v$ through the pullback $f^{*}(g): B \times{ }_{A} C \rightarrow B$ of $g$ along $f$, and then the composite $\left(g^{*}(f)\right) \circ\left(\overline{f^{*}(g)}\right): V \rightarrow C$ yields the needed morphism $w \rightarrow g$ by the universal property of the centralisation functor.

The arrow $W \rightarrow B$ universally induced by $v$ is regular epic, hence so is its restriction $[W, W]_{\mathcal{B}} \rightarrow[B, B]_{\mathcal{B}}$; but a regular quotient of a perfect object is perfect.

Theorem 2.9. Let $\mathcal{A}$ be a semi-abelian category with enough projectives and $\mathcal{B}$ a Birkhoff subcategory of $\mathcal{A}$. An object $A$ of $\mathcal{A}$ is $\mathcal{B}$-perfect if and only if it admits a universal $\mathcal{B}$-central extension. Moreover, this universal $\mathcal{B}$-central extension may be chosen in such a way that it occurs in a short exact sequence

$$
0 \longrightarrow \mathrm{H}_{2}(A, I) \longmapsto \mathrm{U}(A, I) \stackrel{u_{A}^{I}}{\longrightarrow} A \longrightarrow 0 .
$$

Proof. If an object admits a universal $\mathcal{B}$-central extension then it is $\mathcal{B}$-perfect by Lemma 1.8. Conversely, let $f: B \rightarrow A$ be a weakly universal central extension of a $\mathcal{B}$-perfect object $A$ (Lemma 2.4). Then by Lemma 2.7 it admits a (weakly universal central) subobject with a $\mathcal{B}$-perfect domain. By Proposition 1.9, this subobject is also universal. The shape of the short exact sequence follows from 2.5 .

Proposition 2.10. Let $\mathcal{A}$ be a semi-abelian category with enough projectives and $\mathcal{B}$ a Birkhoff subcategory of $\mathcal{A}$. If $f: B \rightarrow A$ is a $\mathcal{B}$-central extension with a $\mathcal{B}$-perfect domain $B$, then $f$ is a quotient of a universal $\mathcal{B}$-central extension.

Proof. The construction in the proof of Theorem 2.9 may be adapted to the given extension $f$ in such a way that the resulting morphism $u \rightarrow f$ is a regular epimorphism. We take a projective presentation $p: P \rightarrow B$ and use the composite $f \circ p: P \rightarrow A$ as a projective presentation of $A$. After centralisation we obtain a weakly universal $\mathcal{B}$-central extension $v: V \rightarrow A$ as in Lemma 2.4 and a regular epic comparison $v \rightarrow f$. Using that $B$ is $\mathcal{B}$-perfect, passing to the perfect subobject as in Lemma 2.8 gives us the needed universal $\mathcal{B}$-central extension $u: U \rightarrow A$ together 
with the induced comparison morphism $v \rightarrow f$. This morphism is still a regular epimorphism by the Birkhoff property of $\mathcal{B}$ (see Subsection 2.1).

2.11. Universal central extensions and abelianisation. It is worth remarking here that a universal $\mathcal{B}$-central extension is always central in an absolute sense, namely, with respect to the abelianisation functor ab: $\mathcal{A} \rightarrow \operatorname{Ab}(\mathcal{A})$. Here $\operatorname{Ab}(\mathcal{A})$ is the Birkhoff subcategory of $\mathcal{A}$ consisting of all objects that admit an internal abelian group structure; see, for instance, [7].

Proposition 2.12. Let $\mathcal{A}$ be a semi-abelian category and $\mathcal{B}$ a Birkhoff subcategory of $\mathcal{A}$. If $f: B \rightarrow A$ is a $\mathcal{B}$-central extension with a $\mathcal{B}$-perfect domain $B$, then $f$ is $\mathrm{Ab}(\mathcal{A})$-central. In particular, universal $\mathcal{B}$-central extensions are always $\operatorname{Ab}(\mathcal{A})$ central.

Proof. We have $B \cong[B, B]_{\mathcal{B}}$ since $B$ is $\mathcal{B}$-perfect; $[B, B]_{\mathcal{B}} \cong\left[B \times_{A} B, B \times_{A} B\right]_{\mathcal{B}}$ because $f$ is $\mathcal{B}$-central. Hence the diagonal $B \rightarrow B \times_{A} B$, being isomorphic to $\mu_{B}:\left[B \times_{A} B, B \times_{A} B\right]_{\mathcal{B}} \rightarrow B \times_{A} B$, is a kernel. By Proposition 3.1 in [7, this implies that $f$ is $\operatorname{Ab}(\mathcal{A})$-central. Finally, if $f: B \rightarrow A$ is a universal $\mathcal{B}$-central extension then $B$ is $\mathcal{B}$-perfect.

\section{Nested Birkhoff subcategories}

We now consider the situation where a Birkhoff subcategory $\mathcal{B}$ of a semi-abelian category $\mathcal{A}$ has a further Birkhoff subcategory $\mathcal{C}$ so that they form a chain of nested semi-abelian categories with enough projectives, $\mathcal{C} \subset \mathcal{B} \subset \mathcal{A}$. For instance, $\mathcal{C}$ could be $\operatorname{Ab}(\mathcal{A})$ as in Theorem 4.4 below. Then there is a commutative triangle of left adjoint functors (all right adjoints are inclusions):

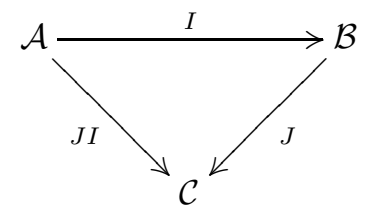

Lemma 3.1. Under the given circumstances,

(1) an object of $\mathcal{B}$ is $J$-perfect if and only if it is $J I$-perfect;

(2) an extension in $\mathcal{B}$ is $J$-central if and only if it is JI-central;

(3) an extension of $\mathcal{A}$ is $\mathcal{B}$-central as soon as it is $J I$-central.

Proof. If $B$ is an object of $\mathcal{B}$ then $J(B)=J I(B)$, which proves the first statement. As for the second statement, an extension $f: B \rightarrow A$ in $\mathcal{B}$ is $J$-central if and only if the square in the diagram on the left
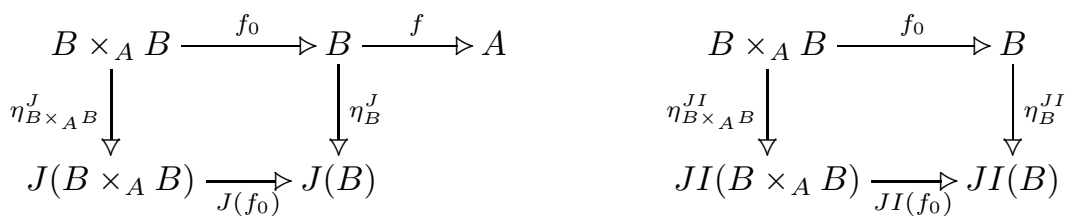

is a pullback in $\mathcal{B}$. Now the inclusion of $\mathcal{B}$ into $\mathcal{A}$ preserves and reflects all limits and moreover $J\left(f_{0}\right)=J I\left(f_{0}\right)$, so that $f$ being $J$-central is equivalent to $f$ being $J I$-central. The third statement follows from the fact that $I$ preserves the pullback on the right above for any $J I$-central extension $f$ in $\mathcal{A}$.

Lemma 3.2. For any object $B$ of $\mathcal{B}$, the reflection from $\mathcal{A}$ to $\mathcal{B}$ restricts to an adjunction

$$
\operatorname{Centr}_{J I}(B) \stackrel{I}{\stackrel{\perp}{\longleftarrow}} \operatorname{Centr}_{J}(B)
$$


Hence the functor I preserves universal central extensions:

$$
I\left(u_{B}^{J I}: \mathrm{U}(B, J I) \rightarrow B\right) \cong\left(u_{B}^{J}: \mathrm{U}(B, J) \rightarrow B\right),
$$

for any $J$-perfect object $B$.

Proof. First of all, by Lemma 3.1,2, $\operatorname{Centr}_{J}(B) \subset(\mathcal{B} \downarrow B)$ is a subcategory of $\operatorname{Centr}_{J I}(B) \subset(\mathcal{A} \downarrow B)$.

Suppose that $g: C \rightarrow B$ is a $J I$-central extension. Applying the functor $I$, we obtain the extension $I(g)=g \circ \eta_{C}^{I}: I(C) \rightarrow B$, which is $J I$-central as a quotient of $g$. Being an extension in $\mathcal{B}, I(g)$ is $J$-central by Lemma 3.12 .

Finally, as any left adjoint functor, $I$ preserves initial objects.

Proposition 3.3. Suppose that $\mathcal{C} \subset \mathcal{B} \subset \mathcal{A}$ is a chain of inclusions of Birkhoff subcategories (with the left adjoints written as in (D) of a semi-abelian category $\mathcal{A}$. If $B$ is a J-perfect object of $\mathcal{B}$ then we have the exact sequence

$$
0 \longrightarrow[\mathrm{U}(B, J I), \mathrm{U}(B, J I)]_{\mathcal{B}} \longmapsto \mathrm{H}_{2}(B, J I) \longrightarrow \mathrm{H}_{2}(B, J) \longrightarrow 0,
$$

and $u_{B}^{J I}=u_{B}^{J}$ if and only if $[\mathrm{U}(B, J I), \mathrm{U}(B, J I)]_{\mathcal{B}}$ is zero.

Proof. By Lemma 3.2 and Theorem 2.9, if $B$ is a $J$-perfect object of $\mathcal{B}$ then the comparison morphism between the induced universal central extensions gives rise to the $3 \times 3$ diagram in Figure 1 .

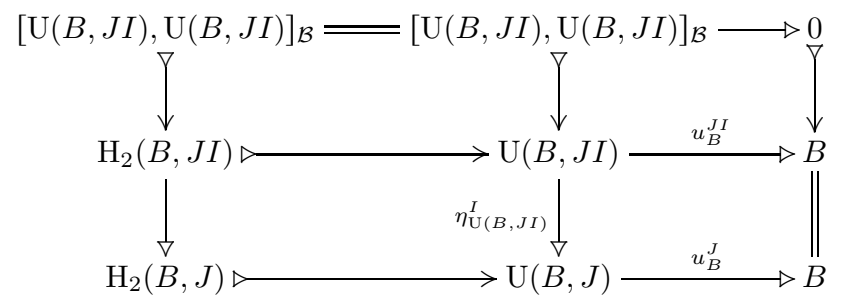

Figure 1. Proof of Proposition 3.3

\section{The Universal CEnTRAL EXtension CONDITION}

We now prove a classical recognition result for universal $\mathcal{B}$-central extensions. To do so, we shall need that $\mathcal{A}$ satisfies the universal central extension condition (UCE) (see Definition 4.1 below). We show that this condition is not only sufficient but in some sense also necessary (Proposition 4.5). We shall moreover ask that $\mathcal{B}$ contains $\operatorname{Ab}(\mathcal{A})$, so that we may suitably reduce the given situation to the case of abelianisation. The examples in 5.1 explain why these conditions are not automatically satisfied. The main result we work towards is Theorem 4.4, which says that a $\mathcal{B}$-central extension $u: U \rightarrow A$ is universal if and only if $\mathrm{H}_{1}(U, I)=\mathrm{H}_{2}(U, I)=0$.

Definition 4.1. Let $\mathcal{A}$ be a semi-abelian category with enough projectives. We say that $\mathcal{A}$ satisfies condition (UCE) when the following holds: if $B$ is an $\operatorname{Ab}(\mathcal{A})$ perfect object, and $f: B \rightarrow A$ and $g: C \rightarrow B$ are $\operatorname{Ab}(\mathcal{A})$-central extensions, then the extension $f \circ g$ is $\operatorname{Ab}(\mathcal{A})$-central.

Lemma 4.2. Let $\mathcal{A}$ be a semi-abelian category with enough projectives satisfying condition (UCE) and $\mathcal{B}$ a Birkhoff subcategory of $\mathcal{A}$ that contains $\operatorname{Ab}(\mathcal{A})$. If $u: U \rightarrow A$ is a $\mathcal{B}$-central extension and $v: V \rightarrow U$ is a universal $\mathcal{B}$-central extension then the extension $u \circ v$ is $\mathcal{B}$-central. 
Proof. By Proposition 2.12 both $u$ and $v$ are $\operatorname{Ab}(\mathcal{A})$-central. Moreover, since $\operatorname{Ab}(\mathcal{A})$ is contained in the Birkhoff subcategory $\mathcal{B}$ of $\mathcal{A}$, the objects $U, V$ and $A$ are $\operatorname{Ab}(\mathcal{A})$ perfect. Now by condition (UCE), the composite $u \circ v: V \rightarrow A$ is also $\operatorname{Ab}(\mathcal{A})$-central. Again using that $\mathcal{B}$ is bigger than $\operatorname{Ab}(\mathcal{A})$ we see that $u \circ v: V \rightarrow A$ is a $\mathcal{B}$-central extension (cf. Lemma 3.1,3).

Under the given assumptions, $u \circ v$ is in fact universal, as shown in Proposition 4.5.

Proposition 4.3. Let $\mathcal{A}$ be a semi-abelian category with enough projectives satisfying condition (UCE) and $\mathcal{B}$ a Birkhoff subcategory of $\mathcal{A}$ that contains $\operatorname{Ab}(\mathcal{A})$. Then in Proposition 1.9, condition 4 implies condition 1. Hence a $\mathcal{B}$-central extension $u: U \rightarrow A$ is universal if and only if its domain $U$ is $\mathcal{B}$-perfect and projective with respect to all $\mathcal{B}$-central extensions.

Proof. Suppose that $u: U \rightarrow A$ is a universal $\mathcal{B}$-central extension; we have to prove that every $\mathcal{B}$-central extension of $U$ splits. By Theorem 2.9, $U$ admits a universal $\mathcal{B}$ central extension $v: V \rightarrow U$. It suffices to prove that this $v$ is a split epimorphism. By Lemma 4.2, the composite $u \circ v$ is $\mathcal{B}$-central. The weak $\mathcal{B}$-universality of $u$ now yields a morphism $s: U \rightarrow V$ such that $u \circ v \circ s=u$. But also $u \circ 1_{U}=u$, so that $v \circ s=1_{U}$ by the $\mathcal{B}$-universality of $u$, and the universal $\mathcal{B}$-central extension $v$ splits. The result follows.

Theorem 4.4. Let $\mathcal{A}$ be a semi-abelian category with enough projectives satisfying condition (UCE) and $\mathcal{B}$ a Birkhoff subcategory of $\mathcal{A}$ containing $\operatorname{Ab}(\mathcal{A}) . A \mathcal{B}$-central extension $u: U \rightarrow A$ is universal if and only if $\mathrm{H}_{1}(U, I)$ and $\mathrm{H}_{2}(U, I)$ are zero.

Proof. $\Rightarrow$ If $u: U \rightarrow A$ is a universal $\mathcal{B}$-central extension then by Proposition 4.3 we have $\mathrm{H}_{1}(U, I)=I(U)=0$ and $U$ is projective with respect to all $\mathcal{B}$-central extensions. This implies that $1_{U}: U \rightarrow U$ is a universal $\mathcal{B}$-central extension of $U$. Theorem 2.9 now tells us that $\mathrm{H}_{2}(U, I)=0$.

$\Leftarrow$ The object $U$ is $\mathcal{B}$-perfect because $I(U)=\mathrm{H}_{1}(U, I)=0$; since $\mathrm{H}_{2}(U, I)$ is also zero, the universal $\mathcal{B}$-central extension $u_{U}^{I}: \mathrm{U}(U, I) \rightarrow U$ of $U$ induced by Theorem 2.9 is an isomorphism. Proposition 4.3 now implies that $U \cong \mathrm{U}(U, I)$ is projective with respect to all $\mathcal{B}$-central extensions. Another application of Proposition 4.3 shows that $u$ is also a universal $\mathcal{B}$-central extension.

Proposition 4.5. Let $\mathcal{A}$ be a semi-abelian category with enough projectives satisfying condition (UCE) and $\mathcal{B}$ a Birkhoff subcategory of $\mathcal{A}$ that contains $\operatorname{Ab}(\mathcal{A})$. Let $f: B \rightarrow A$ and $g: C \rightarrow B$ be $\mathcal{B}$-central extensions. Then the composite $f \circ g$ is a universal $\mathcal{B}$-central extension if and only if $g$ is.

Proof. First note that when $g$ is a universal $\mathcal{B}$-central extension then $f \circ g$ is $\mathcal{B}$-central by Lemma 4.2. The central extensions $f \circ g$ and $g$ have the same domain, and by Proposition 4.3 their universality only depends on a property of this domain.

Proposition 4.3 has the following partial converse, which shows that in some sense condition (UCE) is necessary: if we want that conditions 1-5 in Proposition [1.9 are equivalent, independently of the chosen Birkhoff subcategory $\mathcal{B}$ with $\operatorname{Ab}(\mathcal{A}) \subset \mathcal{B} \subset \mathcal{A}$, then the category $\mathcal{A}$ must satisfy condition (UCE). See also Re$\operatorname{mark} 5.3$

Proposition 4.6. Let $\mathcal{A}$ be a semi-abelian category with enough projectives and $\mathcal{B}$ a Birkhoff subcategory of $\mathcal{A}$ containing $\mathrm{Ab}(\mathcal{A})$. If in Proposition [1.9] conditions 1-5 are equivalent, then the following holds: if $B$ is a $\mathcal{B}$-perfect object, and $f: B \rightarrow A$ and $g: C \rightarrow B$ are $\mathcal{B}$-central extensions, then the extension $f \circ g$ is $\mathcal{B}$-central. If, in particular, this happens for $\mathcal{B}=\operatorname{Ab}(\mathcal{A})$, then $\mathcal{A}$ satisfies condition (UCE). 
Proof. Let $B$ be a $\mathcal{B}$-perfect object and consider $\mathcal{B}$-central extensions $f: B \rightarrow A$ and $g: C \rightarrow B$. Let $u: B \rightarrow A$ be a universal $\mathcal{B}$-central extension. Then by Proposition 2.10 the uniquely induced comparison morphism $\bar{f}: u \rightarrow f$ is a regular epimorphism. Pulling back $g$ along $\bar{f}$ as in

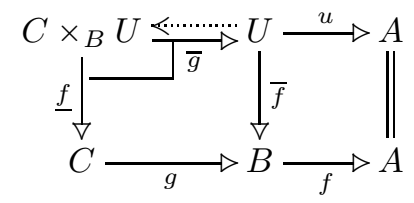

we obtain a splitting for $\bar{g}$ through Proposition 1.9. Now the composite $u \circ \bar{g}$ is a $\mathcal{B}$-central extension because its pullback $u^{*}(u \circ \bar{g})$ along $u$ is a $\mathcal{B}$-trivial extension, as a composite of two $\mathcal{B}$-trivial extensions (Subsection 1.4). Since $f$ is a regular epimorphism by regularity of $\mathcal{A}$, the composite $f \circ g$ is a quotient of the $\mathcal{B}$-central extension $u \circ \bar{g}$, hence is also $\mathcal{B}$-central.

Condition (UCE) allows us to obtain the following refinement of Proposition 3.3 .

Proposition 4.7. Suppose that $\mathcal{A}$ satisfies condition (UCE) and consider a chain of inclusions of Birkhoff subcategories $\operatorname{Ab}(\mathcal{A}) \subset \mathcal{C} \subset \mathcal{B} \subset \mathcal{A}$ with left adjoints as in (D). If $B$ is a J-perfect object of $\mathcal{B}$ then $u_{\mathrm{U}(B, J)}^{J I}=\eta_{\mathrm{U}(B, J I)}^{I}$ and

$$
[\mathrm{U}(B, J I), \mathrm{U}(B, J I)]_{\mathcal{B}} \cong \mathrm{H}_{2}(\mathrm{U}(B, J), J I) .
$$

Hence $u_{B}^{J I}=u_{B}^{J}$ iff $\eta_{\mathrm{U}(B, J I)}^{I}$ is an isomorphism iff $\mathrm{H}_{2}(\mathrm{U}(B, J), J I)$ is zero.

Proof. In view of Remark 2.2, $\eta_{\mathrm{U}(B, J I)}^{I}$ is $J I$-central as a subobject of $u_{B}^{J I}$; hence Proposition 4.5 implies that it is a universal $J I$-central extension of $\mathrm{U}(B, J)$. The result now follows from Theorem 2.9 .

\section{EXAMPLES}

As mentioned in the introduction, our theory is based on the cases of groups (with respect to abelian groups) and Lie algebras over a field $\mathbb{K}$ (with respect to $\mathbb{K}$-vector spaces). It captures results in [14, 21] for $\mathbb{K}$-Leibniz algebras (with respect to $\mathbb{K}$-vector spaces) and gives new results when considering the reflection from Leib $\mathbb{K}_{\mathfrak{K}}$ to $\mathrm{Lie}_{\mathbb{K}}$ (cf. Section 3). The chain of reflections PXMod $\rightarrow$ XMod $\rightarrow$ AbXMod from precrossed modules to crossed modules to abelian crossed modules also corresponds to the situation considered in Section 3. Thus we regain results in [1, 2, 3, 8].

Via Proposition 4.6, the very existence of working theories already shows that in those examples condition (UCE) holds; and it is easy to find further examples. Thus for the rest of the paper we focus on giving counterexamples.

5.1. Two counterexamples. Our first counterexample is borrowed from [9]. It shows that a category - here the category $\mathrm{NAAlg}_{\mathbb{K}}$ of non-associative algebras over a field $\mathbb{K}$, which is a variety of $\Omega$-groups - can be semi-abelian without having to satisfy condition (UCE). This means that NAAlg $\mathbb{K}_{\mathbb{K}}$ does not quite match the picture sketched in Section 4 .

We must also emphasise that condition (UCE) by itself is not yet strong enough to yield results such as Proposition 4.3 or Theorem 4.4 unless $\operatorname{Ab}(\mathcal{A})$ is contained in $\mathcal{B}$. Example 5.4, which explains this, was offered to us by George Peschke. It describes a universal $\mathcal{B}$-central extension $u: U \rightarrow A$ such that $\mathrm{H}_{2}(U, I)$ is not trivial - and indeed one of the assumptions of Theorem 4.4 is violated: the Birkhoff subcategory $\mathcal{B}$ of the (semi-)abelian category $\mathcal{A}$ which we shall consider is strictly smaller than $\operatorname{Ab}(\mathcal{A})$. 
Example 5.2. A non-associative algebra $A$ is a vector space over a field $\mathbb{K}$ equipped with a bilinear operation $[\cdot, \cdot]: A \times A \rightarrow A$. Unlike for Leibniz or Lie algebras (or for associative ones), the bracket need not satisfy any additional conditions. We write $\mathrm{NAAlg}_{\mathbb{K}}$ for the category of non-associative algebras over $\mathbb{K}$ and remark that it coincides with the category of Hom-Leibniz algebras of which the twisting map is trivial ( $\alpha=0$ in the notations of [22, 9]) and with the category of magmas in the monoidal category $\left(\operatorname{Vect}_{\mathbb{K}}, \otimes, \mathbb{K}\right)$. Note that Leib $\mathbb{K}_{\mathbb{K}}$, and hence also Lie $_{\mathbb{K}}$ and Vect $_{\mathbb{K}}$, are subvarieties of NAAlg $\mathbb{K}_{\mathfrak{K}}$. Furthermore, an algebra is abelian if and only if it has a trivial bracket, so precisely when it "belongs to" Vect $\mathbb{K}_{k}$. It is easily seen that an extension $f: B \rightarrow A$ in NAAlg $\mathbb{K}_{\mathbb{K}}$ is Vect $_{\mathbb{K}}$-central if and only if its kernel is contained in the centre of $B$, the object $Z(B)=\{z \in B \mid[z, b]=0=[b, z]$ for all $b \in B\}$.

In [9] the following situation is considered: morphisms $g: C \rightarrow B$ and $f: B \rightarrow A$ where as vector spaces, $A, B$ and $C$ are 2-, 3- and 4-dimensional with respective bases $\left\{a_{1}, a_{2}\right\},\left\{b_{1}, b_{2}, b_{3}\right\}$ and $\left\{c_{1}, c_{2}, c_{3}, c_{4}\right\}$. Their brackets are generated by

$$
\begin{gathered}
{\left[a_{2}, a_{1}\right]=a_{2}, \quad\left[a_{2}, a_{2}\right]=a_{1}} \\
{\left[b_{2}, b_{2}\right]=b_{1}, \quad\left[b_{3}, b_{2}\right]=b_{3}, \quad\left[b_{3}, b_{3}\right]=b_{2}} \\
{\left[c_{3}, c_{2}\right]=c_{1}, \quad\left[c_{3}, c_{3}\right]=c_{2}, \quad\left[c_{4}, c_{3}\right]=c_{4} \quad\left[c_{4}, c_{4}\right]=c_{3}}
\end{gathered}
$$

and zero elsewhere. The algebras $B$ and $A$ are $\operatorname{Vect}_{\mathbb{K}}$-perfect because $[B, B]=B$. The morphism of non-associative algebras $f$ sends $\left(b_{1}, b_{2}, b_{3}\right)$ to $\left(0, a_{1}, a_{2}\right)$ and $g$ sends $\left(c_{1}, c_{2}, c_{3}, c_{4}\right)$ to $\left(0, b_{1}, b_{2}, b_{3}\right)$. The kernel of $f$ is generated by $b_{1}$ and thus equal to the centre $Z(B)$ of $B$. Hence $f$ is $\operatorname{Vect}_{\mathbb{K}}$-central. Likewise, $\operatorname{Ker}(g)$ is generated by $c_{1}$ and thus equal to $Z(C)$, so that $g$ is $V_{e c t}$-central. On the other hand, the kernel of $f \circ g$ contains $c_{2}$, so that $f \circ g$ cannot be Vect $\mathbb{K}_{\mathbb{K}}$-central.

Remark 5.3. Combining Example 5.2 with Proposition 4.6 we obtain a contradiction with the statement of Proposition 6.3 in [10]. It entails the equivalence of all conditions in Proposition 1.9, which would mean that the category of HomLeibniz algebras over $\mathbb{K}$ satisfies condition (UCE). We know, however, that already its subvariety $\mathrm{NAAlg}_{\mathbb{K}}$ does not, which through Lemma 3.1 leads to a clash. It appears that the proof given in [10] does not explain the second half of the "necessary condition".

Example 5.4. Let $C$ be the infinite cyclic group (with its generator written $c \in C$ ) and $R=\mathbb{Z}[C]$ the integral group-ring over $C$. We take $\mathcal{A}$ to be the (abelian) category ${ }_{R}$ Mod of modules over $R$, so that $\operatorname{Ab}(\mathcal{A})=\mathcal{A}$ and condition (UCE) holds. We consider its full subcategory $\mathcal{B}$ of all $R$-modules with a trivial $C$-action; it is clearly Birkhoff in $\mathcal{A}$, and its reflector is determined by tensoring with the trivial $R$-module $\mathbb{Z}$, so that $I(M)=\mathbb{Z} \otimes_{R} M$ for any $R$-module $M$.

Now consider a prime number $p \neq 2$ and let $M$ be the $R$-module $\bigvee_{k \geqslant 1} M_{k}$, where $M_{k}$ for $k \geqslant 1$ is the abelian group $\mathbb{Z}_{p^{k}}=\mathbb{Z} / p^{k} \mathbb{Z}$ equipped with the $C$-action

$$
c \cdot m=(1-p) \cdot m \text {. }
$$

Note that a natural inclusion of $R$-modules $M_{k} \rightarrow M_{k+1}$ is given by

$$
\left(l+p^{k} \mathbb{Z}\right) \mapsto\left(p \cdot l+p^{k+1} \mathbb{Z}\right) .
$$

Then it may be checked that $\mathrm{H}_{2}(M, I)=\mathrm{H}_{2}(C, M) \cong \mathbb{Z}_{p} \neq 0$, while $M$ is $\mathcal{B}$-perfect, and

$$
u: M \rightarrow M: m \mapsto p \cdot m
$$

is a universal $\mathcal{B}$-central extension.

\section{ACKNOWLEDGEMENTS}

We would like to thank the referee, Tomas Everaert, Julia Goedecke and George Peschke for some invaluable suggestions. 


\section{REFERENCES}

[1] D. Arias, J. M. Casas, and M. Ladra, On universal central extensions of precrossed and crossed modules, J. Pure Appl. Algebra 210 (2007), 177-191.

[2] D. Arias, M. Ladra, and A. R.-Grandjeán, Homology of precrossed modules, Illinois J. Math. 46 (2002), no. 3, 739-754.

[3] D. Arias, M. Ladra, and A. R.-Grandjeán, Universal central extensions of precrossed modules and Milnor's relative $K_{2}$, J. Pure Appl. Algebra 184 (2003), 139-154.

[4] F. Borceux and D. Bourn, Mal'cev, protomodular, homological and semi-abelian categories, Math. Appl., vol. 566, Kluwer Acad. Publ., 2004.

[5] D. Bourn, Normalization equivalence, kernel equivalence and affine categories, Category Theory, Proceedings Como 1990 (A. Carboni, M. C. Pedicchio, and G. Rosolini, eds.), Lecture Notes in Math., vol. 1488, Springer, 1991, pp. 43-62.

[6] D. Bourn, $3 \times 3$ Lemma and protomodularity, J. Algebra 236 (2001), 778-795.

[7] D. Bourn and M. Gran, Central extensions in semi-abelian categories, J. Pure Appl. Algebra 175 (2002), 31-44.

[8] P. Carrasco, A. M. Cegarra, and A. R.-Grandjeán, (Co)Homology of crossed modules, J. Pure Appl. Algebra 168 (2002), no. 2-3, 147-176.

[9] J. M. Casas, M. A. Insua, and N. Pachego Rego, On universal central extensions of HomLeibniz algebras, preprint arXiv:1209.6266, 2012.

[10] Y. S. Cheng and Y. C. Su, (Co)homology and universal central extensions of Hom-Leibniz algebras, Acta Math. Sin. (Engl. Ser.) 27 (2011), no. 5, 813-830.

[11] T. Everaert, Higher central extensions and Hopf formulae, J. Algebra 324 (2010), 1771-1789.

[12] T. Everaert and T. Van der Linden, Baer invariants in semi-abelian categories I: General theory, Theory Appl. Categ. 12 (2004), no. 1, 1-33.

[13] T. Everaert and T. Van der Linden, Baer invariants in semi-abelian categories II: Homology, Theory Appl. Categ. 12 (2004), no. 4, 195-224.

[14] A. V. Gnedbaye, Third homology groups of universal central extensions of a Lie algebra, Afrika Math. (Série 3) 10 (1999), 46-63.

[15] M. Gran and T. Van der Linden, On the second cohomology group in semi-abelian categories, J. Pure Appl. Algebra 212 (2008), 636-651.

[16] J. R. A. Gray and T. Van der Linden, Peri-abelian categories and the universal central extension condition, in preparation, 2013.

[17] P. J. Higgins, Groups with multiple operators, Proc. Lond. Math. Soc. (3) 6 (1956), no. 3, 366-416.

[18] G. Janelidze, Galois groups, abstract commutators and Hopf formula, Appl. Categ. Structures 16 (2008), 653-668.

[19] G. Janelidze and G. M. Kelly, Galois theory and a general notion of central extension, J. Pure Appl. Algebra 97 (1994), no. 2, 135-161.

[20] G. Janelidze, L. Márki, and W. Tholen, Semi-abelian categories, J. Pure Appl. Algebra 168 (2002), no. 2-3, 367-386.

[21] J.-L. Loday and T. Pirashvili, Universal enveloping algebras of Leibniz algebras and (co)homology, Math. Ann. 296 (1993), no. 1, 139-158.

[22] A. Makhlouf and S. Silvestrov, Hom-algebra structures, J. Gen. Lie Theory Appl. 2 (2008), no. 2, 51-64.

[23] J. Milnor, Introduction to algebraic K-theory, Princeton University Press, 1972.

[24] Ch. A. Weibel, An introduction to homological algebra, Cambridge Stud. Adv. Math., vol. 38, Cambridge Univ. Press, 1997.

E-mail address: jmcasas@uvigo.es

E-mail address: tim.vanderlinden@uclouvain.be

Dpto. de Matemática Aplicada I, Universidad de Vigo, Escola Enxeñaría Forestal, Campus Universitario A Xunquiera, E-36005 Pontevedra, Spain

Institut de ReCherche en mathématique et physique, Université CATholique de Louvain, Chemin du CyClotron 2 Bte L7.01.02, B-1348 Louvain-LA-Neuve, Belgium

CMUC, University of Coimbra, 3001-454 Coimbra, Portugal 